11. Hattler B, Messenger JC, Shroyer AL, Collins JF, Haugen SJ, Garcia JA, et al; Veterans Affairs Randomized On/Off Bypass (ROOBY) Study Group. OffPump coronary artery bypass surgery is associated with worse arterial and saphenous vein graft patency and less effective revascularization: results from the Veterans Affairs Randomized On/Off Bypass (ROOBY) trial. Circulation. 2012; 125:2827-35.

12. Puskas JD, Williams WH, O'Donnell R, Patterson RE, Sigman SR, Smith AS, et al. Off-pump and on-pump coronary artery bypass grafting are associated with similar graft patency, myocardial ischemia, and freedom from reintervention: long-term follow-up of a randomized trial. Ann Thorac Surg. 2011;91:1836-42; discussion 1842-3.

13. Mohr FW, Morice MC, Kappetein AP, Feldman TE, Ståhle E, Colombo A, et al. Coronary artery bypass graft surgery versus percutaneous coronary intervention in patients with three-vessel disease and left main coronary disease: 5-year follow-up of the randomised, clinical SYNTAX trial. Lancet. 2013;381:629-38.

14. Farkouh ME, Domanski M, Sleeper LA, Siami FS, Dangas G, Mack M, et al; FREEDOM Trial Investigators. Strategies for multivessel revascularization in patients with diabetes. $N$ Engl J Med. 2012;367:2375-84

15. Lamy A, Devereaux PJ, Prabhakaran D, Taggart DP, Hu S, Paolasso E, et al; CORONARY Investigators. Off-pump or on-pump coronary-artery bypass grafting at 30 days. $N$ Engl J Med. 2012;366:1489-97.

16. Raja SG, Berg GA. Impact of off-pump coronary artery bypass surgery on systemic inflammation: current best available evidence. J Card Surg. 2007;22: 445-55.

17. Ascione R, Lloyd CT, Underwood MJ, Gomes WJ, Angelini GD. On-pump versus off-pump coronary revascularization: evaluation of renal function. Ann Thorac Surg. 1999;68:493-8.

18. Güler M, Kirali K, Toker ME, Bozbuğa N, Omeroğlu SN, Akinci E, et al. Different CABG methods in patients with chronic obstructive pulmonary disease. Ann Thorac Surg. 2001;71:152-7.

19. Raja SG, Haider Z, Ahmad M. Predictors of gastrointestinal complications after conventional and beating heart coronary surgery. Surgeon. 2003;1:221-8.

20. Houlind K. On-pump versus off-pump coronary artery bypass surgery: what is the status after ROOBY, DOORS, CORONARY and GOPCABE? Future Cardiol. 2013;9:569-79.

21. Takagi H, Umemoto T. All-Literature Investigation of Cardiovascular Evidence (ALICE) Group. Worse long-term survival after off-pump than on-pump coronary artery bypass grafting. J Thorac Cardiovasc Surg. 2014; $148: 1820-9$.

http://dx.doi.org/10.1016/j.jtcvs.2015.03.026

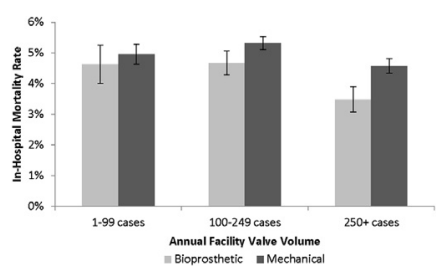

\section{SURGEON VERSUS DEVICE IN AORTIC VALVE REPLACEMENT}

To the Editor:

We believe that inhospital outcomes after valve replacement are at least partially related to the skill of the surgeon, the choice of valve, and the complexities of perioperative management. From a technical perspective, mechanical valve outcomes are likely to be affected by sizing mismatch in larger patients ${ }^{1}$; from a management perspective, mechanical valve outcomes are likely to be affected by the approach to anticoagulation, which is still not well defined. ${ }^{2}$ Surgical and institutional expertise and experience thus still remain important factors affecting outcomes of mechanical versus bioprosthetic valves.

Our study was based on the national cohort and included all patients who received bioprosthetic and mechanical aortic valves. Data on mortality and

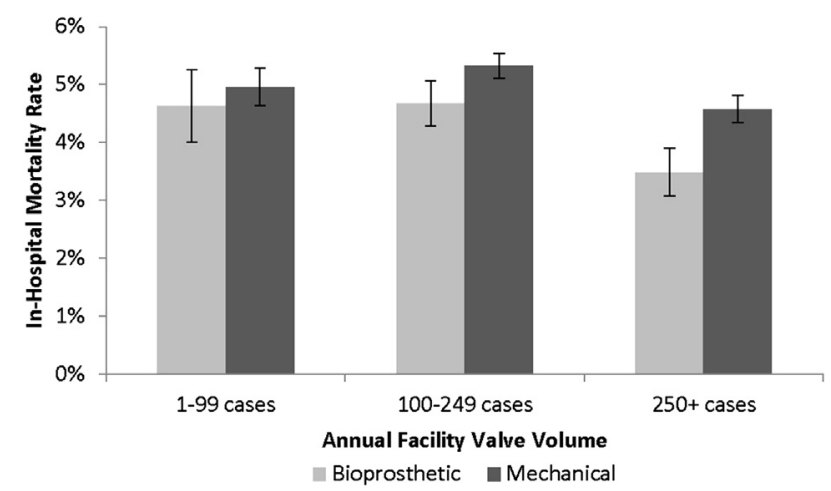

FIGURE 1. In-hospital mortalities for mechanical and bioprosthetic aortic valve replacements according hospital valve volume, with patients matched by propensity score within each hospital. Patients were selected from the Nationwide Inpatient Sample in the years 1998 through 2011. Error bars represent the $95 \%$ confidence interval.

complications, such as thromboembolism, were included for each treatment modality. We found increased inhospital mortality associated with mechanical valves relative to bioprosthetic valves, even after adjustment for all known confounders using propensity scores. Further, when patients were propensity score matched within each center, the difference in mortality persisted.

Hospitals with lower volumes were more likely to use mechanical valves, and it is possible that quality of care in these hospitals is an unmeasured variable. The accompanying editorial written by a respected expert focuses on this aspect of the evidence. ${ }^{4}$ The editorial highlights that surgeon expertise, volume, and anticoagulation protocol for mechanical and biologic valves are important and can serve as surrogate markers for quality of medical care and higher mortality in institutions with lower volumes, including the "failure to rescue" for complications. We believe, however, that persistence of findings after propensity score matching within each center is evidence that factors beyond the facilities' quality of care levels are responsible for the higher mortality associated with mechanical valves (Figure 1). Even if we assume that there are no other factors, one can still argue that this can also be a device safety issue. When the device technology is complex and requires proper protocols for sizing, placement and management that are more likely to be well implemented in institutions with higher volumes, should we really separate the effect of the device safety from delivery of care? The device manufacturers and regulators need to be aware of the issues affecting implant performance under usual care, and we believe that the complexity of care and the device should be viewed as a package from a device safety and effectiveness perspective.

We also recognize that the safety issues might be related to some specific mechanical valves and not to all devices. The absence of unique device identifiers makes it extremely difficult, if not impossible, to assess manufacturer-specific product performance in the real-world setting. ${ }^{6}$ The Society 
of Thoracic Surgeons recently started detailed data collection on devices; the information is incomplete, however, and it will take a long time to achieve enough data and statistical power to detect small differences in valve outcomes. In the interim, we should consider collection of explants and evaluation of autopsy reports to get more information on causes of death and to provide key information for decision making for regulators, patients, and surgeons.

Art Sedrakyan, $M D, P h D$

Abby Isaacs, $M S$

Jeffrey Shuhaiber, $M D$

New York-Presbyterian Hospital

Weill Cornell Medical College

New York, NY clinical outcomes. ${ }^{3}$ It is certainly possible that the evolution of devices has rendered earlier prospective, randomized control trials of mechanical versus prosthetic valves irrelevant. However, if it is the valve (or the requisite technical or medical aspects of valve selection) that is specifically driving the observed difference in mortality, then the difference should be reflected specifically in the valverelatedness of the mortalities observed. In the absence of any such signal, the distinction between valve selection and quality of care will await more convincing data. However, with the emerging success and increasing application of transcutaneous aortic valve replacement, the question may well remain unanswered.

Paul Kurlansky, MD

Division of Surgery

Columbia University

New York, NY

\section{References}

1. Grady D, Herrington D, Bittner V, Blumenthal R, Davidson M, Hlatky M, et al; HERS Research Group. Cardiovascular disease outcomes during 6.8 years of hormone therapy: Heart and Estrogen/progestin Replacement Study follow-up (HERS II). JAMA. 2002;288:49-57.

2. Loomba RS, Arora R. Statin therapy and aortic stenosis: a systematic review of the effects of statin therapy on aortic stenosis. Am J Ther. 2010;17:e110-4.

3. Murphy GJ, Pike K, Rogers CA, Wordsworth S, Stokes EA, Angelini GD, et al. Liberal or restrictive transfusion after cardiac surgery. N Engl J Med. 2015;372: 997-1008.

http://dx.doi.org/10.1016/j.jtcvs.2015.05.021

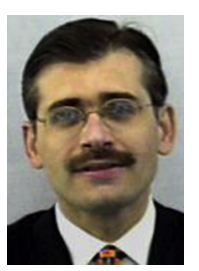

\section{EPIDURALS, INTRAOPERATIVE FLUID ADMINISTRATION, AND HYPOTENSION}

To the Editor:

I enjoyed the recent article, "Effect of the amount of intraoperative fluid administration on postoperative pulmonary complications following anatomic lung resections" by Arslantas and colleagues ${ }^{1}$ in the January issue of Journal of Thoracic and Cardiovascular Surgery.

Our group agrees that all patients undergoing anatomic pulmonary resection should have limited fluid administration because it decreases the number of postoperative pulmonary complications. We have seen substantially greater amounts of fluid administration in patients who receive epidural catheters for pain control, and in recent years have decreased our use of epidural catheters for this reason. Our impression is that the reduced fluid administration as well as the reduced incidence of intraoperative and postoperative hypotension decreases postoperative complication rates.

The study by Arslantas and colleagues ${ }^{1}$ noted that epidural use was not significantly different between patients 